\title{
ArtigoOrigne
}

\section{HISTEROSSONOSSALPINGOGRAFIA E HISTEROSSALPINGOGRAFIA NO DIAGNÓSTICO DE PERMEABILDADE TUBÁRIA EM PACIENTES INFÉRTEIS}

\author{
I. deAlmgda, C. Souza, F. Reginatto, J .S. Cunha Filmo, A. Fagn, F. Fretas, Y. Lamc, *E. P. Passos \\ Segir-Serviço deEcografia, G enética eReprodução Humana - HospitaldeC línicasdePorto Alegre- \\ UniversidadeFederaldo Rio G randedo Sul, Porto Alegre, RS
}

RESUMO - OBJETIVOS. Comparar a histerossonossalpingografia (HSS) em relação à histerossalpingografia (HSG) na avaliação de fator tubário em pacientes inférteis.

Material e Método. Foi realizado um estudo transversal com 30 pacientesem investigação de infertilidade, com idade inferior a 38 anos. As pacientes foram submetidas à HSS, HSG e videolaparoscopia (LPC) na primeira fase do ciclo menstrual. Foi comparada a avaliação de permeabilidade tubária da HSS e da HSG, utilizando-se a LPC como padrão-ouro. Foi calculado 0 valor preditivo negativo (VP-) dos exames. A HSS utilizou como meio de contraste 0 Ecovist ${ }^{\oplus}$, a HSG utilizou contraste iodado hidrossolúvel e a LPC utilizou a cromotubagem com azul de metileno. Foi considerada como diferença estatisticamente significativa um $p<0,05$.
Resultados. A amostra final de estudo foi de 26 pacientes (quatro abandonaram a investigação). $A$ idade média da amostra foi de 30,6 anos. A HSS demonstrou permeabilidade tubária em pelo menos uma das tubas em 24 pacientes $(92,3 \%)$. A HSG demonstrou permeabilidade em uma das tubas em $25(96,2 \%)$, e a LPC em 25 pacientes (96,2\%) 0 VP. da HSS foi de $92 \%$ e o VP. da HSG foi de $100 \%$. N ão houve diferença estatística significante entre a HSS e a HSG ( $p=0,996$, teste de Yates).

Conclusōes. A HSS é um método alternativo à HSG na avaliação de permeabilidade tubária de pacientes inférteis.

Unitermos. Permeabilidade tubária. Histerossalpingografia. Ecografia transvaginal. Infertilidade.

\section{INTRODUÇão}

0 fator tuboperitonealéumaimportante causa de infertilidade feminina. Sua freqüênciacomo causa de infertilidadefemininano Hospital de C línicas de Porto Alegre (HCPA) éde 50\% (Passosetal. 1997)1. 0 diagnóstico de fator tuboperitoneal foi iniciado por Rubin, em 1920, com o uso de dióxido de carbono parainvestigar permeabilidade tubária2. Pouco tempo depois, a histerossalpingografia (HSG) tornou-se o método maisutilizado, principalmenteapós a introdução de meios iodados hidrossolúveis (Mitriet al. 1991)3.

0 utrosmétodossurgiram após eforam

*Correspondência:

R. Ramiro Barcelos, 2350 -Cep: $90035-003$ Porto A legre/ RS-Tel.: (51)316-8117 -E-mail: epp@via-rs.net considerados efetivos, seguros, baratos, sem, no entanto, superar a HSG, como 0 teste de Speck, 1948, e o método de Roland et al. (1966) 4.5 .0 método considerado como padrão-ouro para avaliar permeabilidade tubária é a cromotubagem visualizadapelalaparoscopia(LPC) ${ }^{6}$.

A histerossalpingografiaéo método mais utilizado atualmenteparao diagnóstico defator tuboperitoneal por sermaisprático ebarato do que a LPC. Segundo Karande et al. 1995, a HSG possuiumasensibilidade de $65 \%$ euma especificidadede $83 \%$ naavaliação dedoença tubária, com valoresamplamentevariáveissegundo revisão dosmesmosautores?.

A histerossonossalpingografia(HSS) é um exame que utiliza soro fisiológico ou contrastesàbase de galactose e do uso de ultra-som. A HSS surgiuno final da década de 80 como um método capaz de avaliar permeabilidade tubária (D eichert et al. 1989) ${ }^{8}$. Estudos como o de Mitri et al. 1991, demonstraram que a HSS com solução salinaé umaboaopção à $\mathrm{HSG}^{3}$. Peters et al. 1991, Deichertet al. 1992, Volpietal. 1994 revelaram que a HSS pode ser um exametão acurado quanto a HSG $6,9,10$. Até o momento, ostrabalhostêm concordado que a HSSé capaz de avaliar fator tubário, no entanto seu papel na rotinainvestigação aindaédiscutido.

A investigação do fator tubário ébaseadanaHSG, nalaparoscopia, e, mais recentemente, naHSS como método de screening. 0 valor preditivo negativo(VP-) éum importante marcador epidemiológico para a avaliação de um método em estudo, pois evidencia a probabilidade de um exame 
negativo excluir adoença.

Paraavaliar o papel daHSSem comparação à HSG na rotina de avaliação da pacienteinfértil, foi realizado um estudo prospectivo que comparou o valor preditivo negativo da endossonografia e da histerossonossalpingografianaavaliação de fator tubário em relação àlaparoscopia no setor de Reprodução Humana do HCPA.

\section{Material e Método}

Foi realizado um estudo transversal, duplo-cego, em 30 pacientes inférteis do setor de Reproduçãa H umanado Hospital de Clínicas de Porto Alegre no período de abril adezembro de 1998. Foram incluídas no estudo todas as pacientes que estivessem iniciando a investigação por infertilidade em nosso serviço duranteeste período. Foram excluídas as pacientescomidade superior a 38 anos, com históriade cirurgia tubáriapréviacomo salpingectomia, salpingostomiaou salpingoplastia, ouquese recusassem aassinar ostermo de consentimento do estudo. As pacientes foram submetidas a um questionário onde foram avaliados: idade, tipo de infertilidade (primáriaou secundária), e história de cirurgia pélvica prévia. As pacientes foram submetidas à HSG, HSS L LPC na primeirafase do ciclo menstrual.

A HSG foi realizada com contraste iodado hidrossolúvel aquecido, por médicosradiologistas do serviço de radiologiado HCPA, einterpretadapor médicos daequipe de reprodução assistida. As pacientes não utilizaram qualquer medicação durante o exame. A histerossalpingografiafoi consideradaanormal se não houvesse passagem de contraste por uma e/ou duas tubas uterinas, assim como na presença de hidrossalpinge.

A HSSfoi realizadanaprimeirametade do ciclo menstrual. Umasondade duas vias (Foley número 10) foi inserida no canal

Tabela 1 - Distribuição da alterações da HSS na amostra

\begin{tabular}{lcl}
\hline HSS & Alterada & Normal \\
Útero & $4(15,4)$ & $22(84,6)$ \\
Trompadireita & $5(19,2)$ & $21(80,8)$ \\
Trompa esquerda & $7(26,9)$ & $19(73,1)$ \\
\hline
\end{tabular}

Tabela 2 - Distribuição da alterações da HSG na amostra

\begin{tabular}{lcc}
\hline HSG & Alterada & Normal \\
Útero & $3(11,5)$ & $23(88,5)$ \\
Trompadireita & $2(7,7)$ & $24(92,3)$ \\
Trompa esquerda2 $(11,5)$ & $23(88,5)$ & \\
\hline
\end{tabular}

cervical, ocluindo o colo uterino. Foi realizadaaultra-sonografiatransvaginal eapós término deste exame inicial foi injetado 20 $\mathrm{ml}$ de Ecovist ${ }^{\circledR}$ pela sonda, e observado 0 enchimento da cavidade uterina, difusão do contraste pelas tubas e acúmulo no fundo de saco de D ouglas. Foi utilizado um ecógrafo AlokaSSD 500, com transdutor transvaginal de $5 \mathrm{MHz} .0$ examefoiconsiderado normal quando foi observada difusão do contraste pelastubas ou acúmulo no fundo desaco de Douglas.

A laparoscopia foi realizada sob anestesia geral. As pacientes foram submetidas a punção para introdução de $\mathrm{CO}_{2}$ e formaçãa de pneumoperitônio. $\mathrm{A}$ seguir, introduzia-se o trocarte para a passagem de endoscópio com diâmetro de $10 \mathrm{~mm}$ e realizava-se a segunda punção auxiliar para colocar novo trocarte com $5 \mathrm{~mm}$ de diâmetro, através do qual passava-se um pal pador para auxiliar 0 exame da pelve. A pós a visão da pelve, procedia-se a cromotubagem com azul de metileno e observação do extravasamento do contraste através dos óstios tubários peritoneais. A laparoscopia era considerada alterada quando não houvesse passagem de azul de metileno à cromotubagem e/ou na presença de hidrossalpinge. 0 s médicos que realizaram a laparosco pia não conheciam o resultado da HSG e da HSS, e viceversa. 0 estudo foi aprovado pela comissão de ética Grupo de Pesquisa e Pós-G raduação do H ospital de Clínicas de Porto Alegre.

0 sdadosforam analisados no programa EPInfo versão 6.02 e PEPI. Foram calculados valores preditivos negativos dosexamesem relação à LPC, para determinar a probabilidade do exame excluir obstrução tubária. 0 nível de significância foi de $5 \%$, para o teste de Yates. Foi considerada como diferença estatisticamente significativa um $p<0,05$.

\section{Resultados}

A amostra inicial de 30 pacientes foi reduzidaa26 pacientes, poistrês pacientes abandonaram ainvestigação (doissem causaaparente eum por descobrir-se portadora do vírus da imunodeficiência humana adquirida), e um pacienterecusou-se arealizar aHSS. As 26 pacientes realizaram os trêsexames. 
Tabela 3- Comparação entre HSS e LPC na amostra

\begin{tabular}{lcc}
\hline LPC alterada & LPC & N ormal \\
HSS alterada & 0 & 2 \\
HSS normal & 1 & 23 \\
\hline
\end{tabular}

Valor preditivo negativo $92 \%$
Tabela 4 - Comparação entre HSG e LPC na amostra

\begin{tabular}{lcc}
\hline LPC alterada & LPC & Normal \\
HSG alterada & 1 & 0 \\
HSG normal & 0 & 25 \\
\hline
\end{tabular}

Valor preditivo negativo $100 \%$

A idade média da amostra foi de 30,6 anos. Em média, as pacientes realizaram os exames no $8^{\circ}$ dia do ciclo com um desvio padrão de dois dias. $\mathrm{Na}$ amostra, 23 (88,5\%) pacientes não possuíam cirurgia pélvica préviae 23 pacientes $(88,5 \%$ ) tratavam-se de casos de infertilidade primária. $N$ ão ocorreram complicações dos exames realizados.

A HSSdemonstrou permeabilidade tubáriaem pelo menosumadastrompas em $24(92,3 \%)$ pacientese obstrução bilateral em dois $(7,7 \%)$ pacientes. 0 sresultadosda HSS quanto à alteraçãao uterina ealteração por trompauterinaémostradanatabela1. A HSG demonstrou permeabilidade em pelo menos uma das trompas em 25 (96,2\% ) pacientese obstrução bilateral em um (3,8\%) paciente. 0 sresultadosdaH SG são mostradosnatabela2.

A LPC demonstrou permeabilidadeem pelo menos uma das trompas em 25 (96,2\%) pacientese obstrução bilateral em 1(3,8\%) paciente. A LPC revelou 23 $(88,5 \%)$ de casos com obstrução de trompa direita e $25(96,2 \%)$ de obstrução de trompaesquerda.

A comparação entre HSS e LPC de- monstrou um valor preditivo negativo de 92\% (tabela 3). A comparação entre HSG eLPC demonstrou valor preditivo negativo de $100 \%$ (tabela 4). Estas diferenças não foram estatisticamente significativas. $(p=0.996$ para 0 teste de Yates).

\section{Discussão}

Com o aumento das proporções da doença infamatória pélvica e de suas conseqüências, a avaliação do fator tubário tornou-se muito importante. Com base no exame de triagem, a investigação do fator tubário estaráterminada ou serádirigida paraa LPC. D esta forma, 0 fator mais importante para avaliar um exame diagnóstico é o seu valor preditivo negativo. N osso estudo demonstrou que comparando a HSS com Ecovist ${ }^{\circledR}$ com a HSG na avaliação de permeabilidade tubária, não houve diferença significativa nos seus VP- ou seja, os dois exames possuem o mesmo poder em excluir obstrução tubária e podem ser utilizados para o screening de doença tubária na investigação da infertilidade feminina.

0 s resultados do exame estão em concordânciacom os resultados de Mitri et al. 1991, que determinar am que aHSS éum examesimplesque deve ser utilizado naavaliação preliminar dacavidade uterina etubasuterinas ${ }^{3}$. N este exame, Mitriet al. (1991) demonstraram que os exames possuem concordância diagnóstica em $81 \%$ dos casos para avaliar doença em pelo menos uma das tubas ${ }^{3}$. A avaliação tubária por ultra-sonografia tem se mostrado confiável esatisfatória3,6,7. N aliteratura cita-se uma concordância médiados exames entre 65 e $71 \%$ dos casos (Deichert 1992 et al.) ${ }^{6}$.

U m fator que pode ter ocorrido neste estudo, e citado em vários outros, é a dificuldade no diagnóstico de obstrução uni ou bilateral, baseado somente na visualização do contraste fluindo pelatuba. Isto pode explicar o dados diferentes da HSSem relação àHSG. No entanto, como não houve diferença estística, não se pode dizer que esses números tenham significado. N ossa amostraapresento u um número inferior de casos de fator tubário ao que normalmente éencontrado em nossapopulação.

A HSS mostrou ser um exame com resultados similaresà $\mathrm{HG}$ na avaliação de permeabilidade tubária. Possui aindaalgumas características que a tornam muito atrativa. É um exame que pode ser realizado no consultório pela equipe médica, já na primeira consulta, não necessitando do suporte de um serviço de radiologiacomo a HSG . Não são descritas ocorrências de reações alérgicas, jáque utiliza um meio de contraste à base de galactose (Ecovist); diverso do meio iodado utilizado naHSG. As substâncias contrastadasà base de iodo são comumente utilizadas, preferentemente as hidrossolúveis por possuírem um menor risco de reações alérgicas, no entanto estas reações ainda acontecem (Randolph et al., 1986) ${ }^{11}$. A histerossalpingografiaé um examequeuti- 
lizafluoroscopiadapelve paraavaliação das estruturase pelo menos um filmede raio- $X$ paradocumentar os achados do exame; a dosede radiação média por um exame de 5 a 20 minutos varia entre 1,0 e 3,6 cGy muitas vezes limitando seu uso em infertilidade (Krysiewicz, 1992). ${ }^{12}$ U maoutravantagem daendossonografiaéque ela permite avisualização dosórgãos pélvicos em um maior número deprojeções do que a histerossalpingografia (Krysiewicz, 1992) $)^{12}$.

\section{CONCLUSÃo}

A HSSpermite avaliar a permeabilidade tubáriaem pacientesinférteistendo resultados comparáveis à HSG em excluir fator tubário. Suautilização em serviços deinvestigação de infertilidade proporcionamaior agilidade na seleção de pacientes para a laparoscopiae, ainda, pode ser utilizado em pacientes com contra-indicação ao contrasteiodado.

\section{Summary}

Histerosonosalpingography and Histerosalpingography in the Diagnosis of tubal patency in infertility patients

OBJETIVES. To compare the hysterosonosalpingography(HSS) to the hysterosalpingography(HSG) in the evaluation of the tubal factor in infertility patients.

Material and Methods. A transversal study with 30 patients in infertility investigation, with age minor than 38 years was performed. The patients were submitted to HSS, HSG and laparoscopy (LPC) in the first cycle menstrual phase. The evaluation of tubal patency of HSS and HSG, with LPC like gold-standard was compared. It was calculated the negative predictive value (PV-) of the exams. The HSS used Ecovistò like contrast agent, the HSG used water soluble contrast media and the LPC used tubal insuflation with methylene blue. It was considered significative a $p<0.05$

Results. The final sample was of 26 patients (4 leave the investigation). The mean age was of 30,6 years. The HSS showed tubal patency in at least one of the tubes in 24 patients $(92,3 \%)$. The HSG showed tubal patency in one of the tubes in 25 patients $(96,2 \%)$, and the LPC in 25 patients $(96,2 \%)$. The PV- of theH SS was of $92 \%$ and the PV- of HSG was of $100 \%$. The differences were not statistically significant ( $p=0.996$, Yatestest).

ConcLusions. The HSS is an alternative method in the evaluation of tubal patency in infertility patients. [Rev Ass Med Bras $46(4) ; 342-5]$

UnITERMS:Tubal patency. Hysterosalpingography. Transvaginal ultrasonography. Infertility.

\section{ReferênCias Biblográficas}

1. Passos EP, Freitas FF, Facin AC, C unha Filho JSL. Infertilidade e técnicas de reprodução assistida. In: Freitas FF, Menke CH, Rivoire W A, Passos EP. Rotinas em Ginecologia. 3 ed. Porto Alegre. Ed. Artes Médicas, 1997; 317-326.

2. Rubin IC, N on-operative determination of patency ofFallopian tubesin sterility. A preliminary report. JAM A 1920; 74: 1017-1022.

3. Mitri FF, Andronikou AD, Perpinyal S, H of- meyer G], Sonnendecker EWW. A clinical comparision of sonographic hydrotubation and hysterosapingography. $\mathrm{Br} J$ Obstet Gynecol 1991;98:1031-1036.

4. Speck G. Revision of the PSP (Speck) test for tubal patency. Am J Obstet Gynecol 1948; 55; 1048-1053.

5. Roland M, Clyman A, D ecker A. Further studies on the value of water soluble media in hysterosalpingography. Fertil Steril 1966; 17: 605-612.

6. Deichert U, Schlief R, Sandt M, D aume E. Transvaginal hysterosalpingo-contrast sonography for the assessment of tubal patency with gray scale imaging and additioned use of pulsed wave D oppler. Fertil Steril 1992; 57: 62-67.

7. Karande VC, Pratt DE, Rabin DS, Gleicher $\mathrm{N}$. The limited value of hysterosalpingography in assessing tubal status and fertility potencial. Fertil Steril 1995; 63: 11671171.

8. D eichert $U$, Schlief $R$, Van de Sandt $M$, Juhnke I. Transvaginal hysterosalpingo-contrast sonography (Hy-Co-Sy) compared with conventional tubal diagnosis. H um Reprod 1989; 5: 418-424.

9. Peters A, Coulam CB. Hysterosalpingography with color doppler ultrasonography. Am J Obstet Gynecol 1991; 164: 1530-1534.

10. Volpi E, Grandis T, Rusticheli S, Zuccaro G, Patriarca A, Sismondi P. A new technique to test tubal patency under transvaginal sonographic control. Acta Obstet Gynecol Scand 1994; 73: 797-801.

11. RandolfJR, Ying YK, Maier DE, Schmidt $C L$, Riddik DH. Comparison of real type ultrasonography, histerosalpingography, and laparoscopy, hysteroscopy in the evaluation of uterine abnormalities and tubal patency. Fertil Steril 1986; 46:828-32.

12. Krysiewicz S. Infertility in women: Diagnostic evaluation with hysterosalpingography and other imaging techniques. AJR 1992; 159: 253-261.

Artigo recebido: 17/11/99

Aceito para publicação: 06/04/00 